Article

\title{
Continuous-Service M/M/1 Queuing Systems
}

\author{
Song Chew \\ Department of Mathematics and Statistics, Southern Illinois University Edwardsville, Edwardsville, IL 62026, \\ USA; schew@siue.edu; Tel.: +1-618-650-3016
}

Received: 29 March 2019; Accepted: 24 April 2019; Published: 28 April 2019

\begin{abstract}
In this paper, we look into a novel notion of the standard $\mathrm{M} / \mathrm{M} / 1$ queueing system. In our study, we assume that there is a single server and that there are two types of customers: real and imaginary customers. Real customers are regular customers arriving into our queueing system in accordance with a Poisson process. There exist infinitely many imaginary customers residing in the system. Real customers have service priority over imaginary customers. Thus, the server always serves real (regular) customers one by one if there are real customers present in the system. After serving all real customers, the server immediately serves, one at a time, imaginary customers residing in the system. A newly arriving real customer presumably does not preempt the service of an imaginary customer and hence must wait in the queue for their service. The server immediately serves a waiting real customer upon service completion of the imaginary customer currently under service. All service times are identically, independently, and exponentially distributed. Since our systems are characterized by continuous service by the server, we dub our systems continuous-service $\mathrm{M} / \mathrm{M} / 1$ queueing systems. We conduct the steady-state analysis and determine common performance measures of our systems. In addition, we carry out simulation experiments to verify our results. We compare our results to that of the standard $\mathrm{M} / \mathrm{M} / 1$ queueing system, and draw interesting conclusions.
\end{abstract}

Keywords: queueing systems; simulation; operations research; optimization

\section{Introduction and Motivation}

"I wonder approximately how long I'll have to wait in line." This thought has gone through all our minds as we joined a queue to wait at the bank, the fast food restaurant, or any other place that required waiting for service. For the most part, we do not know the answer to this question but in queuing theory, mathematical models for queues are developed so that this and other questions may be answered. The study of queues has been rigorously carried out since the early twentieth century. Erlang is among the pioneer researchers in queueing theory in the early twentieth century. Erlang [1] studies the operations of telephone systems. The commonly used notation for queueing systems $(a / b / c$ where $a$ describes the arrival process, $b$ indicates the service process, and $c$ specifies the number of servers) is first proposed by Kendall and Morse [2,3] writes the first textbook on the subject of queues. Applications of queueing models may be widely found in traffic control, manufacturing and production systems, healthcare, etc.

Researchers often employ queueing models to study traffic flow. For example, in [4], Kerbache and Macgregor use an expansion method on queueing networks to study traffic control. Heidemann utilizes queueing theory to approximate the queue length and waiting time at intersections [5-7]. Plans and scheduled transportation vehicle fleet in a congested traffic environment [8].

Queueing theory is widely applied in the study of manufacturing and production systems. For example, see [9-11] for the way queueing techniques are employed to analyze manufacturing systems. Most recently, a host of researchers actively investigate into a variation, $\mathrm{M}^{[X]} / \mathrm{G}(a, b) / c$ systems, of the standard M/G/1 system. Batch arrivals and machine repairs are often imposed on these systems; hence, results obtained from their studies are most applicable to production systems; see, for example, [12-17]. 
Research in effective healthcare delivery and access commonly uses queueing analysis. For example, Worthington employs queueing models to study waiting times in hospitals [18], and Christie and Levary use simulation in planning the transportation of patients to hospitals following disasters [19]. In [20], Harper and Shahani model the utilization of beds in hospitals. On the other hand, a queueing approach is employed to manage medical appointments and to identify good nursing levels [21,22]. The reference [23] may provide a further review on the use of queueing models in healthcare operations.

The standard $\mathrm{M} / \mathrm{M} / 1$ system is a queuing system where the interarrival and service times are independently and exponentially distributed with a single server. In the standard M/M/1 system, the server serves customers one by one when customers exist in the system. The server becomes idle when there are not any customers present in the system. When a customer arrives into the system during an idle period, the customer enters into the service immediately since there is no queue. However, during a busy period, a new customer arriving into the system does not preempt the current active service and hence must wait in the queue for their service. In this paper we present a novel notion of the standard $\mathrm{M} / \mathrm{M} / 1$ queueing system.

We call the systems under consideration the continuous-service $\mathrm{M} / \mathrm{M} / 1$ queueing system. The continuous-service $\mathrm{M} / \mathrm{M} / 1$ system is different from the standard system in that the server is continuously serving, and is therefore never idle. To manifest the aforementioned characteristic, we assume that there are two types of customers in this system: real customers and imaginary customers. We further assume that there are an infinite number of imaginary customers already present in the queue, but real customers arrive into the system according to a Poisson process. An arriving real customer into the system does not preempt the current service of a (real or imaginary) customer and therefore needs to join the queue. Real customers have service priority over imaginary customers. Hence, upon service completion of a customer, the server will always first attend to a real customer over an imaginary one if there is at least one real customer waiting in queue; an imaginary customer is only and always (since infinitely many imaginary customers waiting in queue and hence rendering continuous service) attended to if there are no real customers waiting in queue. Note that service times for real customers and that for imaginary customers follow the same exponential distribution. As follows, we provide two motivating examples that may be modeled using our systems.

- A receptionist answers phone calls when there is no customer physically present to attend to. The switchboard is always busy and there are an infinite number of calls waiting (imaginary customers). The receptionist will have to finish answering a call before attending to a customer who physically shows up during the call service (real customers). The receptionist only gets to answer calls when no customer physically waits in queue;

- An industrial robotic arm sorts a number of types of parts, determining which belongs to what group by using an overhead camera for assessment before each action. One may in this case consider that one particular type of part (real customers) has priority over the other type of part (imaginary customers).

In this paper, we conduct the steady-state analysis of, and determine common performance measures of the continuous-service $\mathrm{M} / \mathrm{M} / 1$ queueing system. In addition, we carry out simulation experiments to verify our results. We compare our results to that of the standard $\mathrm{M} / \mathrm{M} / 1$ system, and draw interesting conclusions.

The remainder of the paper is organized as follows. Section 2 derives the steady-state distribution of the continuous-service $\mathrm{M} / \mathrm{M} / 1$ queueing system. Section 3 determines the average number of customers in the system and the average number of customers in the queue; Section 4, on the other hand, studies the system and queue time distributions. Finally, we offer concluding remarks in Section 5 . 


\section{Derivation of the Steady-State Distribution}

This section seeks to derive the steady-state distribution of the continuous-service M/M/1 queueing system. The model of our continuous-service $M / M / 1$ systems and that of the standard $M / M / 1$ system share many similarities. In the meantime, they do differ greatly in many aspects. Figure 1 depicts the birth-death model of our systems. We describe our notation in the following. The state of a system refers to the number of real customers residing in the system. As shown in Figure 1, a system may be in a positive state or a negative state. Most notably, a system is in a positive state if the server is currently serving a real customer. On the contrary, a system is in a non-positive state if the server is currently serving an imaginary customer. More specifically, let $N$ represent the number of real customers in a system. Then, that $N=0$ means that there is no real customers in the system; note, however, that the server must currently be serving an imaginary customer (infinitely many imaginary customers in system to ensure continuous-service of the server upon emptying of all real customers in system). When $N=1$, this means that there is one real customer in the system with 0 in the queue and 1 in service; when $N=-1$, this, on the other hand, means that there is 1 real customer in the system with the 1 in queue while an imaginary customer currently at service (a newly arriving real customer into the system does not preempt the current service of an imaginary customer and hence must wait in queue). If $N=2$, this means that there are 2 real customers in the system with 1 in queue and 1 in service; if $N=-2$, this means that there are 2 real customers in the system with the 2 in queue while an imaginary customer currently at service. In general, that $N=j$ indicates that there are $j$ real customers in the system with $j-1$ in queue and 1 in service, and that $N=-j$ implies that there are $j$ real customers in the system with the $j$ in queue while an imaginary customer is currently at service, $j=$ $1,2, \ldots$. The state $N=0$ simply stands for the fact that there is not any real customer in the system but an imaginary customer is currently being served. Subsequently, we let $\pi_{j}=P(N=j)$, the probability that the system is in state $j, j=\ldots,-2,-1,0,1,2, \ldots$; in other words, $\pi_{j}$ is the probability that there are $j$ real customers present in the system accordingly. In addition, the following are also being employed:

$\lambda$ : Exponential arrival rate of real customers into the system,

$\mu$ : Exponential service rate of the server for all customers, real and imaginary,

$\rho$ : Traffic intensity of the system with $\rho=\frac{\lambda}{\mu}$ and $0 \leq \rho<1$ [24].

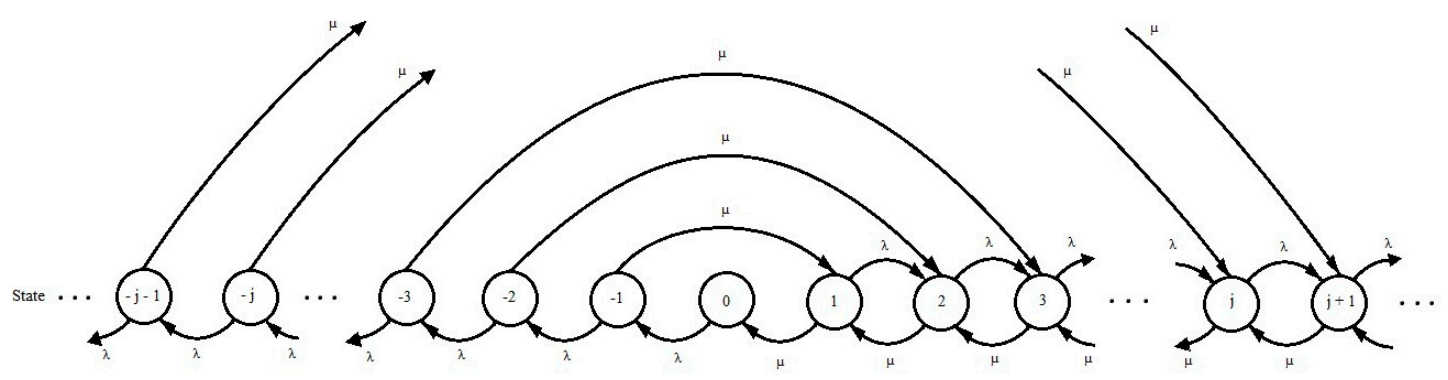

Figure 1. A rate diagram for the continuous-service $M / M / 1$ queueing system.

We now derive the steady-state distribution $\pi=\left\{\ldots, \pi_{-2}, \pi_{-1}, \pi_{0}, \pi_{1}, \pi_{2}, \ldots\right\}$ for our system. To this end, we work out the flow balance equations by making a cut as shown in Figure 2 and employing the notion of flow balance where net flow into a cut must equal net flow out of the cut [24].

We now proceed to write the flow balance equations. For the cuts on the left side of State 0 , we have:

(Between States 0 and -1$): \lambda \pi_{0}=\mu\left(\pi_{-1}+\pi_{-2}+\pi_{-3}+\cdots\right)$

as shown in Figure 2.

Subsequently,

(Between States -1 and -2$): \lambda \pi_{-1}=\mu\left(\pi_{-2}+\pi_{-3}+\pi_{-4}+\cdots\right)$ 


$$
\begin{gathered}
\text { (Between States }-2 \text { and }-3): \begin{array}{c}
\lambda \pi_{-2}=\mu\left(\pi_{-3}+\pi_{-4}+\pi_{-5}+\cdots\right) \\
\vdots
\end{array} \\
\text { (Between States }-j \text { and }-j-1): \lambda \pi_{-j}=\mu\left(\pi_{-j-1}+\pi_{-j-2}+\pi_{-j-3}+\cdots\right)
\end{gathered}
$$

For the cuts on the right side of State 0, we have

$$
\text { (Between States } 0 \text { and } 1): \mu \pi_{1}=\mu\left(\pi_{-1}+\pi_{-2}+\pi_{-3}+\cdots\right)
$$

(Between States 1 and 2$): \mu \pi_{2}=\lambda \pi_{1}+\mu\left(\pi_{-2}+\pi_{-3}+\pi_{-4}+\cdots\right)$

(Between States 2 and 3) $: \mu \pi_{3}=\lambda \pi_{2}+\mu\left(\pi_{-3}+\pi_{-4}+\pi_{-5}+\cdots\right)$

(Between States $j$ and $j+1): \mu \pi_{j}=\lambda \pi_{j-1}+\mu\left(\pi_{-j}+\pi_{-j-1}+\pi_{-j-2}+\cdots\right)$.

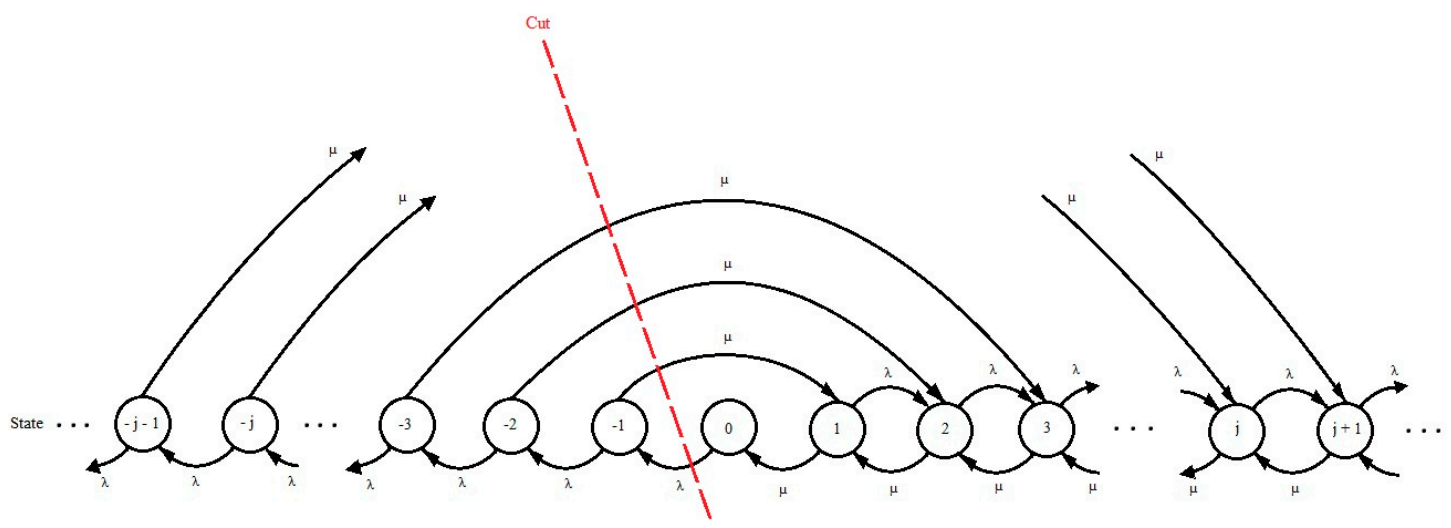

Figure 2. A rate diagram for the continuous-service $M / M / 1$ queueing system with a cut.

Combining Equations (1) and (2), we may see that

$$
\begin{gathered}
\mu \pi_{1}=\lambda \pi_{0} \\
\mu \pi_{2}=\lambda \pi_{1}+\lambda \pi_{-1} \\
\mu \pi_{3}=\lambda \pi_{2}+\lambda \pi_{-2} \\
\vdots \\
\mu \pi_{j}=\lambda \pi_{j-1}+\lambda \pi_{-(j-1)}
\end{gathered}
$$

Adding up Equations in (3) yields

$$
\mu\left(\pi_{1}+\pi_{2}+\pi_{3}+\pi_{4}+\cdots\right)=\lambda\left(\pi_{0}+\pi_{1}+\pi_{-1}+\pi_{2}+\pi_{-2}+\pi_{3}+\pi_{-3}+\cdots\right) .
$$

Dividing through by $\mu$ gives us

$$
\pi_{1}+\pi_{2}+\pi_{3}+\pi_{4}+\cdots=\rho \cdot 1
$$

recall that $\rho=\frac{\lambda}{\mu}$ and that total probabilities sum to 1 .

Thus,

$$
\pi_{1}+\pi_{2}+\pi_{3}+\pi_{4}+\cdots=\rho .
$$


We now seek to express $\pi_{-j}$ in terms of $\pi_{0}$. To this end, we divide Equations (1) through by $\mu$ to produce

$$
\begin{aligned}
\pi_{0} & =\frac{1}{\rho}\left(\pi_{-1}+\pi_{-2}+\pi_{-3}+\cdots\right) \\
& =\frac{1}{\rho} \pi_{-1}+\frac{1}{\rho}\left(\pi_{-2}+\pi_{-3}+\pi_{-4}+\cdots\right) .
\end{aligned}
$$

We may also obtain $\pi_{-1}=\frac{1}{\rho}\left(\pi_{-2}+\pi_{-3}+\pi_{-4}+\cdots\right)$.

Thus,

$$
\begin{aligned}
\pi_{0} & =\frac{1}{\rho} \pi_{-1}+\pi_{-1} \\
& =\left(1+\frac{1}{\rho}\right) \pi_{-1},
\end{aligned}
$$

and hence

$$
\pi_{-1}=\left(\frac{\rho}{1+\rho}\right) \pi_{0}
$$

Similarly,

$$
\begin{aligned}
\pi_{-1} & =\frac{1}{\rho}\left(\pi_{-2}+\pi_{-3}+\pi_{-4}+\cdots\right) \\
& =\frac{1}{\rho} \pi_{-2}+\frac{1}{\rho}\left(\pi_{-3}+\pi_{-4}+\pi_{-5}+\cdots\right) \\
& =\frac{1}{\rho} \pi_{-2}+\pi_{-2} \\
& =\left(1+\frac{1}{\rho}\right) \pi_{-2},
\end{aligned}
$$

which gives us

$$
\begin{aligned}
\pi_{-2} & =\left(\frac{\rho}{1+\rho}\right) \pi_{-1} \\
& =\left(\frac{\rho}{1+\rho}\right)^{2} \pi_{0} .
\end{aligned}
$$

Likewise for $\pi_{-2}$,

$$
\begin{aligned}
\pi_{-2} & =\frac{1}{\rho}\left(\pi_{-3}+\pi_{-4}+\pi_{-5}+\cdots\right) \\
& =\frac{1}{\rho} \pi_{-3}+\frac{1}{\rho}\left(\pi_{-4}+\pi_{-5}+\pi_{-6}+\cdots\right) \\
& =\frac{1}{\rho} \pi_{-3}+\pi_{-3} \\
& =\left(1+\frac{1}{\rho}\right) \pi_{-3},
\end{aligned}
$$

which gives rise to

$$
\begin{aligned}
\pi_{-3} & =\left(\frac{\rho}{1+\rho}\right) \pi_{-2} \\
& =\left(\frac{\rho}{1+\rho}\right)^{3} \pi_{0} .
\end{aligned}
$$

As we continue in this manner to solve for each successive $\pi_{-j}$, we may see a trend that gives rise to the following in terms of $\pi_{0}$,

$$
\pi_{-j}=\left(\frac{\rho}{1+\rho}\right)^{j} \pi_{0}
$$

We are now in a position to determine the value of $\pi_{0}$ in the following. Since total probabilities sum to 1, Equation (4) results in

$$
\pi_{0}+\pi_{-1}+\pi_{-2}+\pi_{-3}+\cdots=1-\rho .
$$

Combining Equations (9) and (10) yields

$$
\begin{array}{r}
\pi_{0}+\left(\frac{\rho}{1+\rho}\right) \pi_{0}+\left(\frac{\rho}{1+\rho}\right)^{2} \pi_{0}+\left(\frac{\rho}{1+\rho}\right)^{3} \pi_{0}+\cdots=1-\rho \\
\pi_{0}\left[1+\left(\frac{\rho}{1+\rho}\right)+\left(\frac{\rho}{1+\rho}\right)^{2}+\left(\frac{\rho}{1+\rho}\right)^{3}+\cdots\right]=1-\rho .
\end{array}
$$

The infinite series in the square brackets above is a geometric series, so we may write it as

$$
\pi_{0}\left(\frac{1}{1-\frac{\rho}{1+\rho}}\right)=1-\rho \pi_{0}(1+\rho)=1-\rho .
$$


Thus,

$$
\pi_{0}=\frac{1-\rho}{1+\rho} .
$$

We proceed to next determine the value of $\pi_{-j}$. Substituting Equation (11) into Equation (9) returns

$$
\begin{aligned}
& \pi_{-1}=\left(\frac{\rho}{1+\rho}\right)\left(\frac{1-\rho}{1+\rho}\right) \\
&=\frac{\rho(1-\rho)}{(1+\rho)^{2}} \\
& \pi_{-2}=\left(\frac{\rho}{1+\rho}\right)^{2}\left(\frac{1-\rho}{1+\rho}\right) \\
&=\frac{\rho^{2}(1-\rho)}{(1+\rho)^{3}} \\
& \pi_{-3}=\left(\frac{\rho}{1+\rho}\right)^{3}\left(\frac{1-\rho}{1+\rho}\right) \\
&=\frac{\rho^{3}(1-\rho)}{(1+\rho)^{4}} \\
& \vdots \\
& \pi_{-j}=\left(\frac{\rho}{1+\rho}\right)^{j}\left(\frac{1-\rho}{1+\rho}\right) \\
&=\frac{\rho^{j}(1-\rho)}{(1+\rho)^{j+1}} .
\end{aligned}
$$

Finally, we are now ready to determine the value of $\pi_{j}$. To do this, dividing through Equations (2) by $\mu$, we obtain the following

$$
\begin{gathered}
\pi_{1}=\pi_{-1}+\pi_{-2}+\pi_{-3}+\cdots \\
\pi_{2}=\rho \pi_{1}+\pi_{-2}+\pi_{-3}+\pi_{-4}+\cdots \\
\pi_{3}=\rho \pi_{2}+\pi_{-3}+\pi_{-4}+\pi_{-5}+\cdots \\
\pi_{4}=\rho \pi_{3}+\pi_{-4}+\pi_{-5}+\pi_{-6}+\cdots \\
\vdots \\
\pi_{j}=\rho \pi_{j-1}+\pi_{-j}+\pi_{-j-1}+\pi_{-j-2}+\cdots
\end{gathered}
$$

To simplify $\pi_{1}$ in Equations (13), we see that $\pi_{1}=\rho \pi_{0}$ from Equation (5). Then, the value of $\pi_{1}$ follows from Equation (11),

$$
\pi_{1}=\frac{\rho(1-\rho)}{1+\rho} .
$$

Similarly, we see that $\pi_{2}=\rho \pi_{1}+\rho \pi_{-1}$ from Equations (7). Thus, the value of $\pi_{2}$ follows from Equations (12) and (14),

$$
\begin{gathered}
\pi_{2}=\rho \cdot \frac{\rho(1-\rho)}{1+\rho}+\rho \cdot \frac{\rho(1-\rho)}{(1+\rho)^{2}} \\
=\rho^{2}(1-\rho)\left[\frac{1}{1+\rho}+\frac{1}{(1+\rho)^{2}}\right] \\
=\rho^{2}(1-\rho)\left[\frac{(1+\rho)^{2}-1}{\rho(1+\rho)^{2}}\right] \\
=\rho(1-\rho)\left(1-\frac{1}{(1+\rho)^{2}}\right) .
\end{gathered}
$$


In a similar fashion, we may obtain the following results,

$$
\begin{gathered}
\pi_{3}=\rho^{3}(1-\rho)\left[\frac{1}{1+\rho}+\frac{1}{(1+\rho)^{2}}+\frac{1}{(1+\rho)^{3}}\right] \\
=\rho^{3}(1-\rho)\left[\frac{(1+\rho)^{3}-1}{\rho(1+\rho)^{3}}\right] \\
=\rho^{2}(1-\rho)\left(1-\frac{1}{(1+\rho)^{3}}\right) \\
\pi_{4}=\rho^{4}(1-\rho)\left[\frac{1}{1+\rho}+\frac{1}{(1+\rho)^{2}}+\frac{1}{(1+\rho)^{3}}+\frac{1}{(1+\rho)^{4}}\right] \\
=\rho^{4}(1-\rho)\left[\frac{(1+\rho)^{4}-1}{\rho(1+\rho)^{4}}\right] \\
=\rho^{3}(1-\rho)\left(1-\frac{1}{(1+\rho)^{4}}\right) \\
\quad \vdots \\
\pi_{j}=\rho^{j-1}(1-\rho)\left(1-\frac{1}{(1+\rho)^{j}}\right) .
\end{gathered}
$$

We have now found the steady-state distribution $\pi=\left\{\ldots, \pi_{-2}, \pi_{-1}, \pi_{0}, \pi_{1}, \pi_{2}, \ldots\right\}$ for our continuous-service M/M/1 systems. Piecing together Equations (11), (12), and (15) yields

$$
\pi_{j}=\left\{\begin{array}{lr}
\frac{\rho^{-j}(1-\rho)}{(1+\rho)^{(1-j)}} & \text { for } j=0,-1,-2, \ldots \\
\rho^{j-1}(1-\rho)\left(1-\frac{1}{(1+\rho)^{j}}\right) & \text { for } j=1,2, \ldots
\end{array}\right.
$$

Recall that the steady-state distribution for the standard M/M/1 system is $\pi=\left\{\pi_{0}, \pi_{1}, \pi_{2}, \ldots\right\}$ where $\pi_{j}=\rho^{j}(1-\rho)$ with $j=0,1,2, \cdots$ [25]. Interestingly, our results are similar to that of the standard system but differ largely by some factors.

\section{Derivation of $L$ and $L_{q}$}

This section derives the average number of customers in the system and the average number of customers in the queue for our continuous-service $\mathrm{M} / \mathrm{M} / 1$ queuing systems. These results differ somewhat from that of the standard M/M/1 system. We denote the average number of (real) customers in the system by $L$, and the average number of (real) customers in the queue by $L_{q}$. Recall that we have an infinite number of imaginary customers to attend to for our systems to be continuous. Therefore, we are focused on real customers for our results.

\subsection{The Average Number of Customers in the System}

Our continuous-service $\mathrm{M} / \mathrm{M} / 1$ queuing systems feature the average number of real customers in the system as follows.

$$
L=0 \pi_{0}+1\left(\pi_{1}+\pi_{-1}\right)+2\left(\pi_{2}+\pi_{-2}\right)+3\left(\pi_{3}+\pi_{-3}\right)+\cdots .
$$


Applying Equation (16) yields

$$
\begin{aligned}
& L=1\left[\frac{\rho(1-\rho)}{1+\rho}+\frac{\rho(1-\rho)}{(1+\rho)^{2}}\right]+2\left[\rho(1-\rho)\left(1-\frac{1}{(1+\rho)^{2}}\right)+\frac{\rho^{2}(1-\rho)}{(1+\rho)^{3}}\right]+3\left[\rho^{2}(1-\rho)\left(1-\frac{1}{(1+\rho)^{3}}\right)+\frac{\rho^{3}(1-\rho)}{(1+\rho)^{4}}\right]+\cdots \\
& =(1-\rho)\left[1-\frac{1}{1+\rho}+\frac{\rho}{(1+\rho)^{2}}\right]+2 \rho(1-\rho)\left[1-\frac{1}{(1+\rho)^{2}}+\frac{\rho}{(1+\rho)^{3}}\right]+3 \rho^{2}(1-\rho)\left[1-\frac{1}{(1+\rho)^{3}}+\frac{\rho}{(1+\rho)^{4}}\right]+\cdots \\
& =(1-\rho)\left[1-\frac{1}{(1+\rho)^{2}}\right]+2 \rho(1-\rho)\left[1-\frac{1}{(1+\rho)^{3}}\right]+3 \rho^{2}(1-\rho)\left[1-\frac{1}{(1+\rho)^{4}}\right]+\cdots \\
& =(1-\rho)+2 \rho(1-\rho)+3 \rho^{2}(1-\rho)+\cdots-\frac{(1-\rho)}{(1+\rho)^{2}}-\frac{2 \rho(1-\rho)}{(1+\rho)^{3}}-\frac{3 \rho^{2}(1-\rho)}{(1+\rho)^{4}}-\cdots \\
& =(1-\rho)\left[1+2 \rho+3 \rho^{2}+\cdots-\frac{(1-\rho)}{(1+\rho)^{2}}\left[1+\frac{2 \rho}{1+\rho}+\frac{3 \rho^{2}}{(1+\rho)^{2}}+\cdots\right]\right. \\
& =(1-\rho)\left[\frac{1}{(1-\rho)^{2}}\right]-\frac{(1-\rho)}{(1+\rho)^{2}}\left[\frac{1}{\left(1-\frac{\rho}{1+\rho}\right)^{2}}\right] \\
& =\frac{1}{1-\rho}-(1-\rho) \\
& =\frac{(2-\rho) \rho}{1-\rho}
\end{aligned}
$$

Thus, the average number of real customers in our systems is $L=\frac{(2-\rho) \rho}{(1-\rho)}$. Recall that such a result for the standard $\mathrm{M} / \mathrm{M} / 1$ system is $L=\frac{\rho}{(1-\rho)}$ [25]. Since our continuous-service systems require that a (real) customer arriving into a system currently serving an imaginary customer (no real customers currently in the system) wait in the queue until the current service on the imaginary customer is completed, we may expect the average number of real customers in the system to be larger than that of the standard $\mathrm{M} / \mathrm{M} / 1$ system. This is reflected in our result which is the $L$ from the standard system multiplied by $(2-\rho)$. Hence, our continuous-service value of $L$ is $100(1-\rho) \%$ greater than the standard system value.

\subsection{The Average Number of Customers in the Queue}

The average number of real customers in the queue for our systems is given by

$$
L_{q}=0\left(\pi_{1}+\pi_{0}\right)+1\left(\pi_{2}+\pi_{-1}\right)+2\left(\pi_{3}+\pi_{-2}\right)+3\left(\pi_{4}+\pi_{-3}\right)+\cdots .
$$

Employing Equation (16), we obtain the following.

$$
\begin{aligned}
& L_{q}=1\left[\rho(1-\rho)\left(1-\frac{1}{(1+\rho)^{2}}\right)+\frac{\rho(1-\rho)}{(1+\rho)^{2}}\right]+2\left[\rho^{2}(1-\rho)\left(1-\frac{1}{(1+\rho)^{3}}\right)+\frac{\rho^{2}(1-\rho)}{(1+\rho)^{3}}\right]+ \\
& 3\left[\rho^{3}(1-\rho)\left(1-\frac{1}{(1+\rho)^{4}}\right)+\frac{\rho^{3}(1-\rho)}{(1+\rho)^{4}}\right]+\cdots \\
& =\rho(1-\rho)\left[1-\frac{1}{(1+\rho)^{2}}+\frac{1}{(1+\rho)^{2}}\right]+2 \rho^{2}(1-\rho)\left[1-\frac{1}{(1+\rho)^{3}}+\frac{1}{(1+\rho)^{3}}\right]+ \\
& 3 \rho^{3}(1-\rho)\left[1-\frac{1}{(1+\rho)^{4}}+\frac{1}{(1+\rho)^{4}}\right]+\cdots \\
& =\rho(1-\rho)+2 \rho^{2}(1-\rho)+3 \rho^{3}(1-\rho)+\cdots \\
& =\rho(1-\rho)\left[1+2 \rho+3 \rho^{2}+\cdots\right] \\
& =\rho(1-\rho)\left[\frac{1}{(1-\rho)^{2}}\right] \\
& =\frac{\rho}{(1-\rho)}
\end{aligned}
$$

Hence, the average number of real customers waiting in the queue for our continuous-service systems is given by $L_{q}=\frac{\rho}{1-\rho}$. Notably, our result returns a value greater than that of the standard $\mathrm{M} / \mathrm{M} / 1$ system for which the average number of customers in queue is $L_{q}=\frac{\rho^{2}}{1-\rho}$ [25]. Strikingly, our result equals the average number of customers in system for the standard $\mathrm{M} / \mathrm{M} / 1$ system. All this does not come as a surprise in that every real customers must wait in the queue for our systems because of the nature of continuous service. 
We now compute our theoretical results for $L$ and $L_{q}$ for our systems using Equations (17) and (18), respectively, for $\rho=0.25,0.50$ and 0.75 . We then compare these results to simulated results based upon ARENA simulation runs for 10,000,000 time units. The results are presented in Table 1 below.

Table 1. Comparisons between theoretical and simulated results for $L$ and $L_{q}$.

\begin{tabular}{ccccccc}
\hline & \multicolumn{2}{c}{$\rho=\mathbf{0 . 2 5}$} & \multicolumn{2}{c}{$\rho=\mathbf{0 . 5 0}$} & \multicolumn{2}{c}{$\boldsymbol{\rho} \mathbf{0 . 7 5}$} \\
\cline { 2 - 6 } & Theoretical & Simulated & Theoretical & Simulated & Theoretical & Simulated \\
\hline$L$ & 0.5833 & 0.5832 & 1.5000 & 1.4998 & 3.7500 & 3.7498 \\
$L_{q}$ & 0.3333 & 0.3332 & 1.0000 & 0.9997 & 3.0000 & 2.9998 \\
\hline
\end{tabular}

Table 1 shows that there is a negligible margin of error between the theoretical and simulated results.

\section{The System and Queue Time Distributions}

This section is focused on obtaining the system time and queue time distributions for our continuous-service $\mathrm{M} / \mathrm{M} / 1$ queueing systems. The average time a real customer spends in the system, denoted by $W$; and the average time a real customer spends in the queue, denoted by $W_{q}$, are then determined. The following results are employed to achieve our goal in the subsequent two subsections. Let random variable $S$ represent the service time of a (real or imaginary) customer, where $S \sim \operatorname{EXP}(\mu)$ with density function $s(t)=\mu e^{-\mu t}$, where $t \geq 0$. Then, $P(S \leq t)=1-e^{-\mu t}$, where $t \geq 0$. Let $T=\sum_{i=1}^{n} S_{i}$, where $n=1,2, \ldots$, and $S_{i} \sim \operatorname{EXP}(\mu)$. Then, $T$ follows a Gamma distribution with a shape parameter $n$. Equivalently, $T$ follows an Erlang distribution with a shape parameter $n$ having the following probability density function (PDF) and cumulative distribution function (CDF), respectively,

$$
f(t)=\frac{\mu^{n} t^{n-1} e^{-\mu t}}{(n-1) !}, t \geq 0, \mu>0
$$

and [26],

$$
F(t)=1-\sum_{i=0}^{n-1} e^{-\mu t} \frac{(\mu t)^{i}}{i !}, t \geq 0, \mu>0
$$

\subsection{The System Time Distribution}

We begin with deriving the CDF of the system time for our continuous-service $M / M / 1$ systems. Let $T$ be a random variable representing the amount of time a real customer spends in the system (system time). Recall that $N$ represents the number of real customers in the system. When $N=0$, a new real customer who arrives into the system will have to spend $T=S+S$ (service time for the imaginary customer currently under service plus service time for the said customer) in the system before the said customer leaves the system. When $N=1$, an arriving real customer into the system will have to stay in the system for $T=S+S$ (service time for the real customer currently under service plus service time for the said customer). If $N=2$, then a real customer entering into the system stays for $T=S+S+S$ (service time for the real customer currently under service plus service time for the real customer currently waiting in queue plus service time for the said customer). Then, when $N=3$, we have $T=S+S+S+S$; and so on. Now, we look into $N=-1$, a new real customer arriving into the system will need to stay for $T=S+S+S$ (service time for the imaginary customer currently under service plus service time for the real customer currently waiting in queue plus service time for the said customer) before leaving the system. Similarly, if $N=-2$, we have $T=S+S+S+S$; and so forth.

We employ the Law of Total Probabilities for conditional probabilities to determine the probability that a real customer spends no more than a $t$ amount of time in the system [27]. Employing the 
steady-state distribution of our systems, the results of the Erlang distribution, and the results in Appendix A yields the following.

$$
\begin{aligned}
& P(T \leq t)=P(T=S+S \leq t \mid N=0) P(N=0)+P(T=S+S \leq t \mid N=1) P(N=1)+ \\
& P(T=S+S+S \leq t \mid N=-1) P(N=-1)+P(T=S+S+S \leq t \mid N=2) P(N=2)+ \\
& P(T=S+S+S+S \leq t \mid N=-2) P(N=-2)+ \\
& P(T=S+S+S+S \leq t \mid N=3) P(N=3)+\cdots \\
& =\left[1-\frac{e^{-\mu t}(\mu t)^{0}}{0 !}-\frac{e^{-\mu t}(\mu t)^{1}}{1 !}\right]\left(\pi_{0}+\pi_{1}\right)+\left[1-\frac{e^{-\mu t}(\mu t)^{0}}{0 !}-\frac{e^{-\mu t}(\mu t)^{1}}{1 !}-\frac{e^{-\mu t}(\mu t)^{2}}{2 !}\right]\left(\pi_{-1}+\pi_{2}\right)+ \\
& {\left[1-\frac{e^{-\mu t}(\mu t)^{0}}{0 !}-\frac{e^{-\mu t}(\mu t)^{1}}{1 !}-\frac{e^{-\mu t}(\mu t)^{2}}{2 !}-\frac{e^{-\mu t}(\mu t)^{3}}{3 !}\right]\left(\pi_{-2}+\pi_{3}\right)+\cdots} \\
& =\left(\pi_{0}+\pi_{1}+\pi_{-1}+\pi_{2}+\pi_{-2}+\pi_{3}+\cdots\right)-\frac{e^{-\mu t}(\mu t)^{0}}{0 !}\left(\pi_{0}+\pi_{1}+\pi_{-1}+\pi_{2}+\pi_{-2}+\pi_{3}+\cdots\right)- \\
& \frac{e^{-\mu t}(\mu t)^{1}}{1 !}\left(\pi_{0}+\pi_{1}+\pi_{-1}+\pi_{2}+\pi_{-2}+\pi_{3}+\cdots\right)-\frac{e^{-\mu t}(\mu t)^{2}}{2 !}\left(\pi_{-1}+\pi_{2}+\pi_{-2}+\pi_{3}+\cdots\right)- \\
& \frac{e^{-\mu t}(\mu t)^{3}}{3 !}\left(\pi_{-2}+\pi_{3}+\cdots\right)-\cdots \\
& =1-\frac{e^{-\mu t}(\mu t)^{0}}{0 !}-\frac{e^{-\mu t}(\mu t)^{1}}{1 !}-\frac{e^{-\mu t}(\mu t)^{2}}{2 !}(\rho)-\frac{e^{-\mu t}(\mu t)^{3}}{3 !}\left(\rho^{2}\right)-\cdots \\
& =1-\frac{e^{-\mu t}(\mu t)^{0}}{0 !}-\frac{e^{-\mu t}}{\rho}\left[\frac{(\rho \mu t)^{1}}{1 !}+\frac{(\rho \mu t)^{2}}{2 !}+\frac{(\rho \mu t)^{3}}{3 !}+\cdots\right] \\
& =1-\frac{e^{-\mu t}(\mu t)^{0}}{0 !}-\frac{e^{-\mu t}}{\rho}\left[e^{\rho \mu t}-1\right] \\
& =1-\frac{e^{-\mu t}(\mu t)^{0}}{0 !}-\frac{e^{-(1-\rho) \mu t}}{\rho}+\frac{e^{-\mu t}}{\rho} \\
& =1+\left(\frac{1-\rho}{\rho}\right) e^{-\mu t}-\frac{e^{-(1-\rho) \mu t}}{\rho}
\end{aligned}
$$

Hence, the CDF of the system time $T$ for our continuous-service M/M/1 queuing systems is

$$
F(t)=1+\left(\frac{1-\rho}{\rho}\right) e^{-\mu t}-\frac{1}{\rho} e^{-(1-\rho) \mu t}, \quad t \geq 0, \mu>0,0<\rho<1 .
$$

We may now differentiate Equation (19) to obtain the PDF of the system time $T$ as follows.

$$
f(t)=-\left(\frac{1-\rho}{\rho}\right) \mu e^{-\mu t}+\frac{1}{\rho}(1-\rho) \mu e^{-(1-\rho) \mu t}=\left(\frac{1}{\rho}\right)(1-\rho) \mu e^{-(1-\rho) \mu t}-\left(\frac{1-\rho}{\rho}\right) \mu e^{-\mu t}
$$

Thus, the PDF of the system time $T$ is

$$
f(t)=\left(\frac{1}{\rho}\right)(1-\rho) \mu e^{-(1-\rho) \mu t}-\left(\frac{1-\rho}{\rho}\right) \mu e^{-\mu t}, \quad t \geq 0, \mu>0,0<\rho<1 .
$$

One may be interested in the average time a real customer spends in the system. To this end, we find the expectation of $T$, denoted by $W, W=E(T)=\int_{0}^{\infty}(1-F(t)) d t$ as follows [26].

$$
\begin{aligned}
& W=E(T)=\int_{0}^{\infty}\left[1-\left(1+\left(\frac{1-\rho}{\rho}\right) e^{-\mu t}-\frac{1}{\rho} e^{-(1-\rho) \mu t}\right)\right] d t \\
& =\int_{0}^{\infty}\left(\frac{1}{\rho} e^{-(1-\rho) \mu t}-\left(\frac{1-\rho}{\rho}\right) e^{-\mu t}\right) d t \\
& =\left[-\frac{1}{(1-\rho) \mu \rho} e^{-(1-\rho) \mu t}+\frac{1-\rho}{\mu \rho} e^{-\mu t}\right]_{0}^{\infty} \\
& =[0]-\left[-\frac{1}{(1-\rho) \mu \rho}+\frac{1-\rho}{\mu \rho}\right] \\
& =\frac{1}{\mu \rho}\left(\frac{1}{1-\rho}-\frac{(1-\rho)^{2}}{1-\rho}\right) \\
& =\frac{1}{\lambda}\left(\frac{1-1+2 \rho-\rho^{2}}{1-\rho}\right) \\
& =\frac{(2-\rho) \rho}{\lambda(1-\rho)}
\end{aligned}
$$


Therefore, the average time a real customer spends in system, $W$, is given by $W=\frac{(2-\rho) \rho}{\lambda(1-\rho)}$. We would expect the average time in our systems to be greater than that for the standard $M / M / 1$ system. When we compare our result to that for the standard system, which is $W=\frac{\rho}{\lambda(1-\rho)}$ [25], we can see that our result again is that of the standard system multiplied by $(2-\rho)$. Our result is greater than that of the standard system since our $L$ is greater than that of the standard system. Our result could readily be verified by Little's Law, $L=\lambda W$ [25].

\subsection{The Queue Time Distribution}

We establish the queue time distribution in this subsection. We start off with finding the CDF of the queue time distribution. Let $Y$ be a random variable representing the amount of time a real customer spends in the queue (queue time). Employing similar logic in Section 4.1, we have similar situations as follows. For example, when $N=0$, a new real customer who arrives into the queue will have to spend $Y=S$ (service time for the imaginary customer currently under service) in the queue before the customer enters into service. When $N=1$, an arriving real customer into the queue will have to stay in the queue for $Y=S$ (service time for the real customer currently under service). If $N=2$, then a real customer entering into the queue stays for $Y=S+S$ (service time for the real customer currently under service plus service time for the real customer currently waiting in queue). Then, when $N=3$, we have $Y=S+S+S$; and so on. We now look into $N=-1$, a new real customer arriving into the queue will need to stay for $Y=S+S$ (service time for the imaginary customer currently under service plus service time for the real customer currently waiting in queue) before leaving the queue. Similarly, if $N=-2$, we have $Y=S+S+S$; and so forth.

We again employ the Law of Total Probabilities for conditional probabilities. Likewise, applying the steady-state distribution of our systems, the results of the Erlang distribution, and the results in Appendix A, we work out the probability that a real customer spends less than a $t$ amount of time in the queue as follows.

$$
\begin{aligned}
& P(Y \leq t)=P(Y=S \leq t \mid N=0) P(N=0)+P(Y=S \leq t \mid N=1) P(N=1)+ \\
& P(Y=S+S \leq t \mid N=-1) P(N=-1)+P(Y=S+S \leq t \mid N=2) P(N=2)+ \\
& P(Y=S+S+S \leq t \mid N=-2) P(N=-2)+P(Y=S+S+S \leq t \mid N=3) P(N=3)+\cdots \\
& =\left(1-\frac{e^{-\mu t}(\mu t)^{0}}{0 !}\right)\left(\pi_{0}+\pi_{1}\right)+\left(1-\frac{e^{-\mu t}(\mu t)^{0}}{0 !}-\frac{e^{-\mu t}(\mu t)^{1}}{1 !}\right)\left(\pi_{-1}+\pi_{2}\right)+ \\
& \left(1-\frac{e^{-\mu t}(\mu t)^{0}}{0 !}-\frac{e^{-\mu t}(\mu t)^{1}}{1 !}-\frac{e^{-\mu t}(\mu t)^{2}}{2 !}\right)\left(\pi_{-2}+\pi_{3}\right)+\cdots \\
& =\left(\pi_{0}+\pi_{1}+\pi_{-1}+\pi_{2}+\pi_{-2}+\pi_{3}+\cdots\right)-\frac{e^{-\mu t}(\mu t)^{0}}{0 !}\left(\pi_{0}+\pi_{1}+\pi_{-1}+\pi_{2}+\pi_{-2}+\pi_{3}+\cdots\right)- \\
& \quad \frac{e^{-\mu t}(\mu t)^{1}}{1 !}\left(\pi_{-1}+\pi_{2}+\pi_{-2}+\pi_{3}+\cdots\right)-\frac{e^{-\mu t}(\mu t)^{2}}{2 !}\left(\pi_{-2}+\pi_{3}+\cdots\right)-\cdots \\
& =1-\frac{e^{-\mu t}(\mu t)^{0}}{0 !}-\frac{e^{-\mu t}(\mu t)^{1}}{1 !}(\rho)-\frac{e^{-\mu t}(\mu t)^{2}}{2 !}\left(\rho^{2}\right)-\cdots \\
& =1-e^{-\mu t}\left[1+\frac{(\rho \mu t)^{1}}{1 !}+\frac{(\rho \mu t)^{2}}{2 !}+\cdots\right] \\
& =1-e^{-\mu t}\left[e^{\rho \mu t}\right] \\
& =1-e^{-(1-\rho) \mu t}
\end{aligned}
$$

Hence, the $C D F$ of the queue time $Y$ for our continuous-service $M / M / 1$ queuing systems is

$$
F(t)=1-e^{-(1-\rho) \mu t}, \quad t \geq 0, \mu>0,0<\rho<1 .
$$

To obtain the PDF of the queue time $Y$, we find the derivative of Equation (22), which yields the following PDF.

$$
f(t)=(1-\rho) \mu e^{-(1-\rho) \mu t}, \quad t \geq 0, \mu>0,0<\rho<1
$$


We may now find the expectation of $Y$, the average time a real customer spends in the queue, denoted by $W_{q}$. In fact, Equation (23) is an exponential distribution with parameter $(1-\rho) \mu$. Consequently,

$$
W_{q}=E(Y)=\frac{1}{(1-\rho) \mu}=\frac{\rho}{\lambda(1-\rho)} .
$$

Hence, the average time a real customer spends in queue, $W_{q}$, is

$$
W_{q}=\frac{\rho}{\lambda(1-\rho)}
$$

For similar reason discussed in Section 4.1, we note that our result is greater than that of the standard M/M/1 system, which is $W_{q}=\frac{\rho^{2}}{\lambda(1-\rho)}$ [25], and that our result is equal to the average system time for the standard M/M/1 system. As a matter of fact, Equation (24) may be easily verified by Little's Law, $L_{q}=\lambda W_{q}[25]$.

We now calculate values for $W$ and $W_{q}$ using Equations (21) and (24), respectively, for $\rho=0.25$, 0.50 and 0.75 , and $\mu=10$. We then compare these values to simulated results by ARENA simulation runs for 10,000,000 time units. The results are compiled in Table 2.

Table 2. Comparisons between theoretical and simulated results for $W$ and $W_{q}$.

\begin{tabular}{ccccccc}
\hline & \multicolumn{2}{c}{$\rho=\mathbf{0 . 2 5}$} & \multicolumn{2}{c}{$\rho=\mathbf{0 . 5 0}$} & \multicolumn{2}{c}{$\rho=\mathbf{0 . 7 5}$} \\
\cline { 2 - 7 } & Theoretical & Simulated & Theoretical & Simulated & Theoretical & Simulated \\
\hline$W$ & 0.2333 & 0.2333 & 0.3000 & 0.2999 & 0.5000 & 0.5000 \\
$W_{q}$ & 0.1333 & 0.1333 & 0.2000 & 0.1999 & 0.4000 & 0.4000 \\
\hline
\end{tabular}

As we may see from Table 2, the simulation results for the average system time and average queue time are consistent with the theoretical results, with little to no error at 10,000,000 time units for the simulation runs.

We finally calculate the probabilities that a real customer spends more than 10 time units and more than 20 time units in the system and the probabilities that a real customer spends more than 10 time units and more than 20 time units in the queue. We employ Equations (19) and (22) to determine these probabilities for $\rho=0.25,0.50$ and 0.75 , and $\mu=10$. These are compared to results from ARENA simulation that runs for 100,000,000 time units. Table 3 contains these results.

Table 3. Comparisons between theoretical and simulated results for probabilities that a real customer spends more than 10 time units and more than 20 time units in the system and queue.

\begin{tabular}{lcccccc}
\hline & \multicolumn{2}{c}{$\boldsymbol{\rho}=\mathbf{0 . 2 5}$} & \multicolumn{2}{c}{$\boldsymbol{\rho = \mathbf { 0 . 5 0 }}$} & \multicolumn{2}{c}{$\boldsymbol{0 . 7 5}$} \\
\cline { 2 - 6 } & Theoretical & Simulated & Theoretical & Simulated & Theoretical & Simulated \\
\hline$P(T>10)$ & 0.7858 & 0.7854 & 0.8452 & 0.8448 & 0.9158 & 0.9158 \\
$P(T>20)$ & 0.4865 & 0.4863 & 0.6004 & 0.5997 & 0.7636 & 0.7637 \\
$P(Y>10)$ & 0.4724 & 0.4718 & 0.6065 & 0.6060 & 0.7788 & 0.7790 \\
$P(Y>20)$ & 0.2231 & 0.2227 & 0.3679 & 0.3672 & 0.6065 & 0.6066 \\
\hline
\end{tabular}

Compared to our theoretical results, the simulated probabilities have a negligible margin of error, which we would expect to diminish with longer simulation runs.

\section{Conclusions}

Situations exist in the real world in which we have systems that require continuous service. We noticed this and set out to model these situations in the form of our continuous-service M/M/1 queuing systems. In doing this, we were able to derive and verify by means of simulations, the common 
characteristics of interest for our queuing systems: The average number of real customers in the system and queue, and the average times spent by a real customer in the system and queue. We intuitively compared these values to the standard $\mathrm{M} / \mathrm{M} / 1$ queuing system. It was noticed that the difference between the results of our systems and that of the standard system was by a factor by $(2-\rho)$. We also derived the system time and queue time distributions for our systems and also verified these results with simulations. For any systems that have the server continuously serving, and can be classified as continuous-service $\mathrm{M} / \mathrm{M} / 1$ queuing systems, our results may be leveraged to analyze, estimate, and optimize the systems. Future work may look into analyzing real-world systems that share similar features of our continuous-service $\mathrm{M} / \mathrm{M} / 1$ queuing systems.

Funding: This research received no external funding.

Conflicts of Interest: The author declares no conflict of interest.

\section{Appendix A}

Given the steady-state distribution $\pi=\left\{\ldots, \pi_{-2}, \pi_{-1}, \pi_{0}, \pi_{1}, \pi_{2}, \ldots\right\}$ of our continuous-service $\mathrm{M} / \mathrm{M} / 1$ queueing systems and $0 \leq \rho<1, \sum_{j=n}^{\infty}\left(\pi_{-j}+\pi_{j+1}\right)=\rho^{n}$ for $n=0,1,2, \ldots$

We prove the above identity by induction. We first verify that the above identity holds for $n=0$. In fact the identity follows in that, when $n=0$, we have $\sum_{j=0}^{\infty}\left(\pi_{-j}+\pi_{j+1}\right)=\left(\pi_{0}+\pi_{1}\right)+\left(\pi_{-1}+\pi_{2}\right)+$ $\left(\pi_{-2}+\pi_{3}\right)+\cdots=1=\rho^{0}$ since all probabilities sum to 1 .

We next assume that the identity holds for some positive integer $\mathrm{k}$. That is, we have $\sum_{j=k}^{\infty}\left(\pi_{-j}+\pi_{j+1}\right)=\left(\pi_{-k}+\pi_{k+1}\right)+\left(\pi_{-k-1}+\pi_{k+2}\right)+\left(\pi_{-k-2}+\pi_{k+3}\right)+\cdots=\rho^{k}$.

We finally need to show that the identity holds for $k+1$. That is, we must show that $\sum_{j=k+1}^{\infty}\left(\pi_{-j}+\pi_{j+1}\right)=\left(\pi_{-k-1}+\pi_{k+2}\right)+\left(\pi_{-k-2}+\pi_{k+3}\right)+\cdots=\rho^{k+1}$. To this end, we see that

$$
\begin{aligned}
\sum_{j=k+1}^{\infty}\left(\pi_{-j}+\pi_{j+1}\right) & =\left(\pi_{-k-1}+\pi_{k+2}\right)+\left(\pi_{-k-2}+\pi_{k+3}\right)+\cdots \\
& =\left(\pi_{-k}+\pi_{k+1}\right)+\left(\pi_{-k-1}+\pi_{k+2}\right)+\left(\pi_{-k-2}+\pi_{k+3}\right)+\cdots-\left(\pi_{-k}+\pi_{k+1}\right) \\
& =\rho^{k}-\left(\pi_{-k}+\pi_{k+1}\right) \\
& =\rho^{k}-\frac{\rho^{k}(1-\rho)}{(1+\rho)^{k+1}}-\rho^{k}(1-\rho)\left[1-\frac{1}{(1+\rho)^{k+1}}\right] \text { (Use Equation (16)) } \\
& =\rho^{k}-\rho^{k}(1-\rho)\left[\frac{1}{(1+\rho)^{k+1}}+\left(1-\frac{1}{(1+\rho)^{k+1}}\right)\right] \\
& =\rho^{k}-\rho^{k}(1-\rho) \\
& =\rho^{k+1} .
\end{aligned}
$$

Therefore, the above identity is valid for $n=0,1,2, \ldots$ by induction.

\section{References}

1. Erlang, A.K. Sandsynlighedsregning og telefonsamtaler. Nyt tidsskrift for Matematik 1909, 20, 33-39.

2. Kendall, D.G. Stochastic processes occurring in the theory of queues and their analysis by the method of the imbedded Markov chain. Ann. Math. Stat. 1953, 24, 338-354. [CrossRef]

3. Morse, P.M. Queues, Inventories, and Maintenance; Wiley: New York, NY, USA, 1958.

4. Kerbachea, L.; Smith, J.M. The generalized expansion method for open finite queueing networks. Eur. J. Oper. Res. 1987, 32, 448-461. [CrossRef]

5. Heidemann, D. Queue length and waiting-time distributions at priority intersections. Transp. Res. Part B Methodol. 1991, 25, 163-174. [CrossRef]

6. Heidemann, D. Queue length and delay distributions at traffic signals. Transp. Res. Part B Methodol. 1994, 28, 377-389. [CrossRef]

7. Heidemann, D. A queueing theory approach to speed-flow-density relationships. In Transportation and Traffic Theory, Proceedings of the 13th International Symposium on Transportation and Traffic Theory, Lyon, France, 24-26 July 1996; Elsevier: Oxford, UK, 1996. 
8. Kerbache, L.; Van Woensel, T. Planning and scheduling transportation vehicle fleet in a congested traffic environment. In Supply Chain Management: European Perspectives, 1st ed.; de Koster, M.B.M., Delfmann, W., Eds.; Copenhagen Business School Press: Køge, Denmark, 2005.

9. Groover, M.P. Automation, Production Systems, and Computer-Aided Manufacturing; Prentice Hall: Upper Saddle River, NJ, USA, 1980.

10. Buzacott, J.A.; Shanthikumar, J.G. Stochastic Models of Manufacturing Systems; Prentice Hall: Upper Saddle River, NJ, USA, 1993.

11. Gershwin, S.B. Manufacturing Systems Engineering; Prentice Hall: Upper Saddle River, NJ, USA, 1994.

12. Baba, Y. On the $\mathrm{M}^{[X]} / \mathrm{G} / 1$ queue with vacation time. Oper. Res. Lett. 1986, 5, 93-98. [CrossRef]

13. Krishna Reddy, G.V.; Nadarajan, R.; Arumuganathan, R. Analysis of a bulk queue with $N$ policy multiple vacations and setup times. Comput. Oper. Res. 1998, 25, 957-967. [CrossRef]

14. Choudhury, G. A batch arrival queue with a vacation time under single vacation policy. Comput. Oper. Res. 2002, 29, 1941-1955. [CrossRef]

15. Khalaf, R.F. Queueing systems with four different main server's interruptions and a stand-by server. Int. J. Oper. Res. 2014, 3, 49-54. [CrossRef]

16. Jeyakumar, S.; Senthilnathan, B. Modelling and analysis of a bulk service queueing model with multiple working vacations and server breakdown. RAIRO-Oper. Res. 2017, 51, 485-508. [CrossRef]

17. Ayyappan, G.; Karpagam, S. An $\mathrm{M}^{[\mathrm{X}]} / \mathrm{G}(a, b) / 1$ queueing system with breakdown and repair, stand-by server, multiple vacation and control policy on request for re-service. Mathematics 2018, 6, 101. [CrossRef]

18. Worthington, D.J. Queueing models for hospital waiting lists. J. Oper. Res. Soc. 1987, 38, 413-422. [CrossRef] [PubMed]

19. Christie, P.M.; Levary, R.R. The use of simulation in planning the transportation of patients to hospitals following a disaster. J. Med. Syst. 1998, 22, 289-300. [CrossRef] [PubMed]

20. Harper, P.R.; Shahani, A.K. Modelling for the planning and management of bed capacities in hospitals. J. Oper. Res. Soc. 2002, 53, 11-18. [CrossRef]

21. Green, L.V.; Savin, S. Reducing delays for medical appointments: A queueing approach. Oper. Res. 2008, 56, 1526-1538. [CrossRef]

22. Yankovic, N.; Green, L.V. Identifying good nursing levels: A queuing approach. Oper. Res. 2011, 59, 942-955. [CrossRef]

23. Gupta, D. Queueing models for healthcare operations. In Handbook of Healthcare Operations Management; Springer: New York, NY, USA, 2013.

24. Winston, W.L.; Goldberg, J.B. Operations Research: Applications and Algorithms; Cengage Learning: Boston, MA, USA, 2003.

25. Wagner, H.M. Principles of Operations Research; Prentice Hall: Upper Saddle River, NJ, USA, 1975.

26. Bain, L.J.; Engelhardt, M. Introduction to Probability and Mathematical Statistics; Cengage Learning: Boston, MA, USA, 1991.

27. Wackerly, D.; Mendenhall, W.; Scheaffer, R.L. Mathematical Statistics with Applications; Duxbury Press: Belmont, CA, USA, 2007.

(C) 2019 by the author. Licensee MDPI, Basel, Switzerland. This article is an open access article distributed under the terms and conditions of the Creative Commons Attribution (CC BY) license (http://creativecommons.org/licenses/by/4.0/). 